\title{
Author Correction: nc886 is induced by TGF- $\beta$ and suppresses the microRNA pathway in ovarian cancer
}

\author{
Ji-Hye Ahn ${ }^{1}$, Hyun-Sung Lee (i) ${ }^{2}$, Ju-Seog Lee (i) ${ }^{3}$, Yeon-Su Lee ${ }^{4}$, Jong-Lyul Park ${ }^{5}$, Seon-Young Kim (1) ${ }^{5}$, \\ Jung-Ah Hwang6, Nawapol Kunkeaw ${ }^{7,8}$, Sung Yun Jung ${ }^{9}$, Tae Jin Kim ${ }^{10}$, Kwang-Soo Lee ${ }^{11}$, Sung Ho Jeon ${ }^{11}$, \\ Inhan Lee ${ }^{12}$, Betty H. Johnson" ${ }^{7}$, Jung-Hye Choi $^{1}$ \& Yong Sun Lee (i) ${ }^{7,13}$
}

Correction to: Nat. Commun; https://doi.org/10.1038/s41467-018-03556-7; published online 21 March 2018.

In the original version of the Supplementary Information file associated with this Article, Supplementary Fig. 18 panel b was inadvertently replaced with a duplicate of panel a.

The error has now been fixed and the corrected version of the Supplementary Information PDF is available to download from the HTML version of the Article.

Published online: 19 December 2018

Open Access This article is licensed under a Creative Commons Attribution 4.0 International License, which permits use, sharing, adaptation, distribution and reproduction in any medium or format, as long as you give appropriate credit to the original author(s) and the source, provide a link to the Creative Commons license, and indicate if changes were made. The images or other third party material in this article are included in the article's Creative Commons license, unless indicated otherwise in a credit line to the material. If material is not included in the article's Creative Commons license and your intended use is not permitted by statutory regulation or exceeds the permitted use, you will need to obtain permission directly from the copyright holder. To view a copy of this license, visit http://creativecommons.org/licenses/by/4.0/.

(c) The Author(s) 2018

\footnotetext{
${ }^{1}$ Department of Life and Nanopharmaceutical Sciences and Department of Oriental Pharmaceutical Science, Kyung Hee University, Seoul 02447, Korea. ${ }^{2}$ Division of Thoracic Surgery, Michael E. DeBakey Department of Surgery, Baylor College of Medicine, Houston, TX 77030, USA. ${ }^{3}$ Department of Systems Biology, University of Texas MD Anderson Cancer Center, Houston, TX 77030, USA. ${ }^{4}$ Rare Cancer Branch, Research Institute, National Cancer Center, Goyang 10408, Korea. ${ }^{5}$ Medical Genomics Research Center, KRIBB, Daejeon 34141, Korea. ${ }^{6}$ Genomics Core Laboratory, Omics Core Laboratory, Research Institute, National Cancer Center, Goyang 10408, Korea. ${ }^{7}$ Department of Biochemistry and Molecular Biology, University of Texas Medical Branch, Galveston, TX 77555-1072, USA. ${ }^{8}$ Institute of Molecular Biosciences, Mahidol University, Nakhon Pathom 73170, Thailand. ${ }^{9}$ Verna and Marrs McLean Department of Biochemistry and Molecular Biology, Baylor College of Medicine, Houston, TX 77030, USA. ${ }^{10}$ Department of Obstetrics and Gynecology, Cheil General Hospital and Women's Healthcare Center, College of Medicine Dankook University, Seoul 04619, Korea. ${ }^{11}$ Department of Life Science and Center for Aging and Health Care, Hallym University, Chuncheon 24252, Korea. ${ }^{12}$ miRcore, Ann Arbor, MI 48105, USA. ${ }^{13}$ Department of Cancer Biomedical Science, Graduate School of Cancer Science and Policy, National Cancer Center, Goyang 10408, Korea. Correspondence and requests for materials should be addressed to J.-H.C. (email: jchoi@khu.ac.kr) or to Y.S.L. (email: yslee@ncc.re.kr)
} 